\title{
Genome-wide association study of Parkinson's disease using MAX3 test
}

\author{
Georgii Ozhegov \\ Kazan Federal University, Kazan, \\ Russia \\ Novel Software Systems, Ltd., \\ Novosibirsk, Russia \\ georgii_provisor@mail.ru \\ Dmitry Poverin \\ Novosibirsk State Technical \\ University, Novosibirsk, Russia
}

\author{
Sergey Medvedev \\ Federal Research Center Institute of \\ Cytology and Genetics, Novosibirsk, \\ Russia
}

Suren Zakian

Federal Research Center Institute of Cytology and Genetics, Novosibirsk, Russia

\author{
Yuri Vyatkin \\ Novosibirsk State University, \\ Novosibirsk, Russia \\ Novel Software Systems, Ltd., \\ Novosibirsk, Russia \\ Sergey Postovalov \\ Novosibirsk State Technical \\ University, Novosibirsk, Russia \\ Novosibirsk State University, \\ Novosibirsk, Russia
}

\begin{abstract}
Whole exomes for a set of patients with Parkinson's disease (PD) were sequenced to conduct a genomewide association study (GWAS) using MAX3 test to find novel genomic variants associated with the disease. As a result, several new variants were identified.
\end{abstract}

Key words - Parkinson's disease; GWAS; SNP

\section{Introduction}

One of the priority task of medical genetics is to discover the genetic basis of the etiology and pathogenesis of Parkinson's disease (PD). In this work, we search for known disease markers among patients with confirmed diagnosis of PD and control group. We also conduct a genome-wide association study (GWAS) to identify further common variants that contribute to disease.

\section{Sample preparation}

A sample of 50 patients with diagnosed PD and 10 control individuals was acquired for the study. As a control group, we took examined elderly individuals with no signs of PD and for whom the development of this pathology in the future is considered as unlikely. Whole blood was withdrawn from the patients with further preparation for the sequencing by performing quality check of the libraries and sequencing them on Illumina HiSeq 2500 sequencer in Rapid Run mode. SureSelect XT Target Enrichment System kit was used resulting pair-end reads of 100 b.p. in length.

\section{Data acquisition}

The list of germline single nucleotide polymorphisms (SNPs) was obtained with Genomenal NGSWizard software [1]. Original fastq files were quality checked and mapped to the reference human genome GRCh38/hg38. For SNPs discovery GATK v. 4.1.3.0 software package was used with Best Practice guidelines applied [2]. After SNPs and sample quality control, we acquired genotype data from more than 900 thousands SNPs and short indels. All of them were annotated by SnpEff and Annovar software systems. Among all SNPs we selected around 3000 SNPs appeared in PDassociated genes. PD-associated genes were taken from OMIM, ClinVar and MDSGene databases. Selected SNPs were also analyzed with ClinVar and dbSNP databases. We observed two pathogenic SNPs: one appeared in LRRK2 gene and was found in two patients, another appeared in GLUD2 gene and was found in three patients. It should be emphasized that not a single sample from the cohort with an excluded diagnosis had a pathogenic mutation or variant with an uncertain significance (VUS). Thus, the pathogenic genetic variants for Parkinson's disease were found for 5 out of 50 patients.

\section{Genome-Wide Association Studies}

For further data analyses, we performed a GWAS. We used the MAX3 test to check the hypothesis of an association between Parkinson's disease and biallelic single nucleotide polymorphisms [3]. The MAX3 test is based on the CochranArmitage trend test (CATT). The MAX3 test statistic is calculated as a maximum of the absolute values of CATT statistics for the dominant, recessive, and additive inheritance mode. As a result, the MAX3 test is not much inferior in power to the trend tests in the case of the known inheritance mode, and is robust if the inheritance mode is unknown [4]. To calculate the p-value the MAX3 test the approach proposed in [5] was used. To account for multiple testing, the Bonferroni correction for significance level was performed [6]. As a result, we obtained 83 SNPs in which the p-value is less than 1e-9, while all 10 people from the control group and at least 43 people with Parkinson's disease are present in the sample.

\section{Data analysis}

A fraction of mutations is located on chromosome 17 in the locus where gene $\mathrm{KCNJ} 12$ is located. According to published data, the change of KCNJ12 protein could be involved in PD pathogenesis [7]. In this regard, we additionally analyzed all SNPs from this locus. 264 mutations we observed, but not a single of them was reported in ClinVar database. Most of them were previously analyzed by some projects like 1000genomes, Topmed, GnomAD, ExAC and PAGE. This helped us to determine global alternative allele frequencies for SNPs. Based on this data we isolated suspicious SNPs with high p-values and low global alternative allele frequencies. We state that this combination of signs may indicate pathogenic and associated with PD SNPs. Eventually we identify 10 SNPs with p-value lower than 7e-6 and global alternative allele frequency low than $9 \%$.

\section{Results and discussion}

Around 10 new SNPs associated with PD and not reported previously were identified during the study. Since the sample size was small, we plan to make meta-analysis with results of another GWASes of PD in the future [8]. 


\section{ACKNOWLEDGEMENTS}

The work was supported by RSCF grant No. 19-75-20063.

\section{REFERENCES}

[1] Available at https://ru.genomenal.com

[2] Van der Auwera, Geraldine A., et al. "From FastQ data to highconfidence variant calls: the genome analysis toolkit best practices pipeline." Current protocols in bioinformatics 43.1 (2013): 11-10.

[3] Freidlin, Boris, et al. "Trend tests for case-control studies of genetic markers: power, sample size and robustness." Human heredity 53.3 (2002): 146-152.
[4] Postovalov, Sergey, and R. Wyler Metge. "A power comparison of the association tests for genome-wide association studies." 2016 11th International Forum on Strategic Technology (IFOST). IEEE, 2016.

[5] Zang, Yong, Wing Kam Fung, and Gang Zheng. "Simple algorithms to calculate the asymptotic null distributions of robust tests in case-control genetic association studies in R." Journal of Statistical software 33.8 (2010).

[6] Bonferroni, Carlo E., C. Bonferroni, and C. E. Bonferroni. "Teoria statistica delle classi e calcolo delle probabilita'." (1936).

[7] Tian, Chuan, et al. "Potassium channels: structures, diseases, and modulators." Chemical biology \& drug design 83.1 (2014): 1-26.

[8] Chang, Diana, et al. "A meta-analysis of genome-wide association studies identifies 17 new Parkinson's disease risk loci" Nat Genet. 2017. - Vol. 49(10). P. 1511-1516. 